\title{
Studying the outskirts of reverberation mapped AGNs
}

\author{
Shai Kaspi \\ School of Physics \& Astronomy and the Wise Observatory \\ The Raymond and Beverly Sackler Faculty of Exact Sciences \\ Tel-Aviv University, Tel-Aviv 69978, Israel \\ email: shai@wise.tau.ac.il
}

\begin{abstract}
About 100 AGNs have their black hole mass measured directly using the reverberation mapping technique over the past few decades. By now we have high enough numbers to explore unique subsamples within these objects and to study phenomena across variety of AGNs. I will review recent reverberation mapping studies which focus on high-redshift high-luminosity AGNs and on AGNs with super-Eddington accreting massive black holes. These studies enable to investigate the BLR size, mass, and luminosity relations in different subsamples of AGNs and to check whether there are differences in these relations in different types of AGNs. In particular I will discuss the following questions: Is the BLR size - luminosity relation the same over the whole AGNs luminosity range? Are there different relations for different types of AGNs? What are these studies teaching us about theory of accretion into black holes in AGNs?
\end{abstract}

Keywords. accretion, accretion disks, galaxies: active, galaxies: nuclei

\section{Introduction}

The reverberation mapping (RM) method uses time lags between the emission of broad lines and the continuum in Active Galactic Nuclei (AGNs) in order to determine the distance of the Broad Line Region (BLR) from the central emission source (accretion to the central black hole), and from this to infer the mass of the black hole $(\mathrm{BH})$ in the Center of the AGN (Peterson 1993; Kaspi et al. 2000). RM can also be used to determine the geometry and velocity field of the BLR given sufficiently high resolution spectroscopy over long enough period of time in which the AGN varied and with high cadence of observations.

Over the past three decades almost 100 AGNs with sufficient data to determine their BLR size were studied (see a partial compilation in Bentz \& Katz 2015). This has resulted with a firm determination of a relation between the BLR size $\left(R_{B L R}\right)$ and the AGN luminosity in various wavelength bands (e.g., Kaspi et al. 2005, Bentz et al. 2013). The $R_{B L R}$-Luminosity relation is used together with the width of the broad lines as a proxy to the BLR velocity and some assumptions about the geometry to determine the $\mathrm{BH}$ mass in the center of the AGNs. This yields also a Mass-Luminosity relation for the AGNs.

The $R_{B L R}$-Luminosity relation is broadly used to estimate the $\mathrm{BH}$ mass of thousands of AGNs using a single epoch spectrum of each AGN from which the luminosity and the width of the broad lines are measured. These samples are used for high- and lowluminosity AGNs in order to study the cosmological evolution of AGNs (mass function, accretion history, etc. ; e.g., Vestergaard et al. 2008). However, the measured $R_{B L R}-$ Luminosity relation span only on a limited luminosity range, from $\lambda L_{\lambda}(5100 \AA)$ of $10^{42}$ to $10^{46} \mathrm{erg} \mathrm{s}^{-1}$, and there are hardly no RM measurements outside of that range. In the 


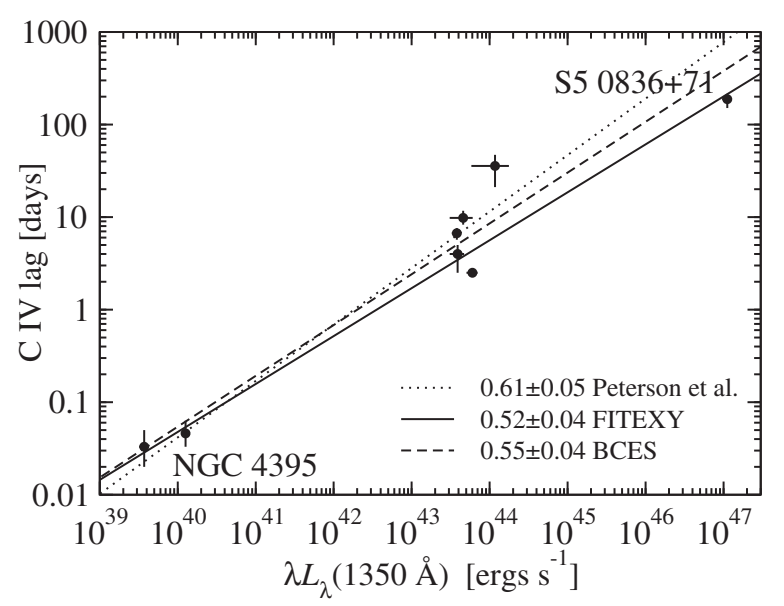

Figure 1. Size-Luminosity relation based on C IV $(1550 \AA)$ emission line and the UV continuum. Data are reproduced from Peterson et al. (2005) to which the result for S5 0836+71 from Kaspi et al. (2007) was added. See Kaspi et al. (2007) for more details. Two more objects are now added to that result and are consistent with the point of S5 $0836+71$.

first part of the this contribution results from a RM campaign of high-luminosity RM campaign will be presented.

With tens of AGNs with measured $R_{B L R}$, studies of subsamples within the AGN phenomenon can now be carried out. In the second part of this contribution results from a RM campaign of Supper Eddington Accreting Massive Black Holes will be described with a comparison to normal accreting AGNs.

\section{Reverberation Mapping of High-Luminosity AGNs}

RM of high-luminosity AGNs (quasars) have not been much successful so far due to several difficulties: RM requires a lot of telescope time for monitoring the AGNs. Highluminosity quasars have much larger $R_{B L R}$ and much longer variability time scales. Thus, a monitoring period of about a decade or more is needed. High-luminosity AGNs are fainter in general and thus a larger telescope is needed. The combination of a large telescope to be dedicated for more than a decade is very hard to to find. The light curves are also smeared due to time dilation and by the large BLR, and thus the response of the line to the continuum variation is very hard to detect.

In 1995 we started a photometric monitoring of 11 high-redshift high-luminosity quasars at the Wise Observatory $1 \mathrm{~m}$ telescope in Israel which is operated by Tel-Aviv University. In 1999 we started to spectroscopically monitor 6 of these quasars at the Hobby-Eberly Telescope (HET) $9 \mathrm{~m}$ telescope which is partly owned by Pennsylvania State University. This yield a RM campaign which lasted for 14 years. The sample ranges on redshift of $2.1<z<3.2$, luminosity of $10^{46}<\lambda L_{\lambda}(5100 \AA)<10^{47.5}$, and the lines that were monitored for each object are two of $\operatorname{Ly} \alpha, \operatorname{C~IV}(1550 \AA)$, and $\mathrm{C} \operatorname{III}(1909 \AA)$, depending on the object's redshift. First preliminary results from the first five years were published by Kaspi et al. (2007; and see Fig. 1).

After 14 years of spectroscopic monitoring and 18 years of photometric monitoring we found that the continuum variation for all object is around the level of $20-50 \%$ and we found time lags of the $\operatorname{CIV}(1550 \AA)$ line in three objects, in one of which we found a time lag for Ly $\alpha$ and for another object we found a time lag of $\mathrm{C} \operatorname{III}(1909 \AA)$. The time lags are all around 200 light days in the rest frame and fall around the preliminary result which 

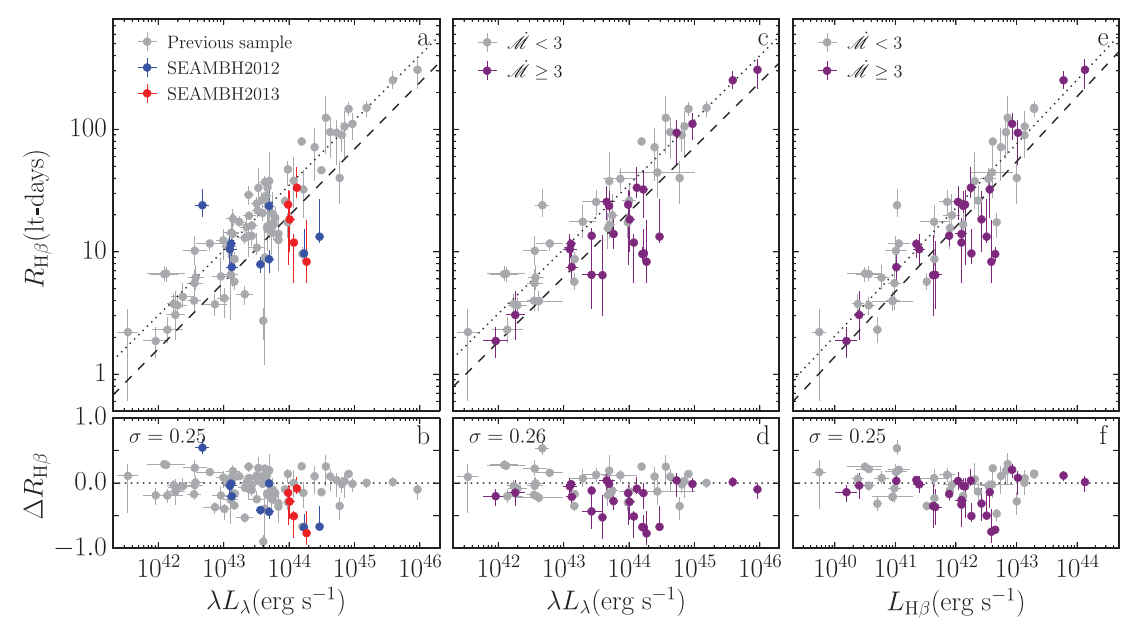

Figure 2. The $R_{B L R}$-Luminosity relations (panels $a, c, e$ ) and the deviations of $R_{B L R}$ from the regression relationships (panel $b, d, f$ ). The dotted line is the fit for the normal AGNs sample whereas the dashed line is the fit for the SEAMBHs sample in all panels (see Du et al. 2015).

is shown in Fig. 1. The relation of $\operatorname{CIV}(1550 \AA)$ size and the UV luminosity seems to be the same over 9 orders of magnitude in luminosity, and high-luminosity AGNs keep the $R_{B L R}$ and Luminosity relation.

\section{Reverberation Mapping of Supper Eddington Accreting Massive Black holes}

Supper Eddington Accreting Massive Black holes (SEAMBHs) are AGNs which accrete into their BHs above their Eddington rate. We carried out a RM campaign with photometric observations from the Wise Observatory $1 \mathrm{~m}$ telescope in Israel and spectroscopic observations from the $2.5 \mathrm{~m}$ telescope in Lijiang observatory in China. Ten AGNs were monitored during 2012 and for nine of then we determined the time lag of the $\mathrm{H} \beta$ line. During 2013 eight AGNs were monitored and for five of them a time lag was determined. The results are presented in the four papers by Wang et al. (2014), Hu et al. (2015), and Du et al. $(2014 ; 2015)$.

One of the main results of this RM campaign is shown in Fig,. 2. The $R_{B L R}$-Luminosity relation for the SEAMBHs lays below this relation for AGNs which are accreting at subEddington rate ("Normal AGNs"). Beyond accretion rate which is about three times the Eddington rate the deviation of individual objects from the $R_{B L R}$-Luminosity relation increases with the accretion rate (higher accretion rate objects have higher deviation from the "Normal" relation). This implies that the $\mathrm{H} \beta$ time lags (BLR size) become shorter (smaller) with increasing accretion rate relative to normal AGNs with the same luminosity.

\section{Slim Accretion Disks Around Supper-Massive BHs}

While thin accretion disk has been suggested to power the $\mathrm{BH}$ in the center of subEddington accreting BHs, Slim accretion disks (which are thicker than thin disks) were suggested to power supper-Eddington accretion disks (e.g. Sodowsky 2011). However, numerical simulations have contradict this paradigm and suggest that radial advection is not important and much of the accretion energy can be released via fast winds. Thus, The 

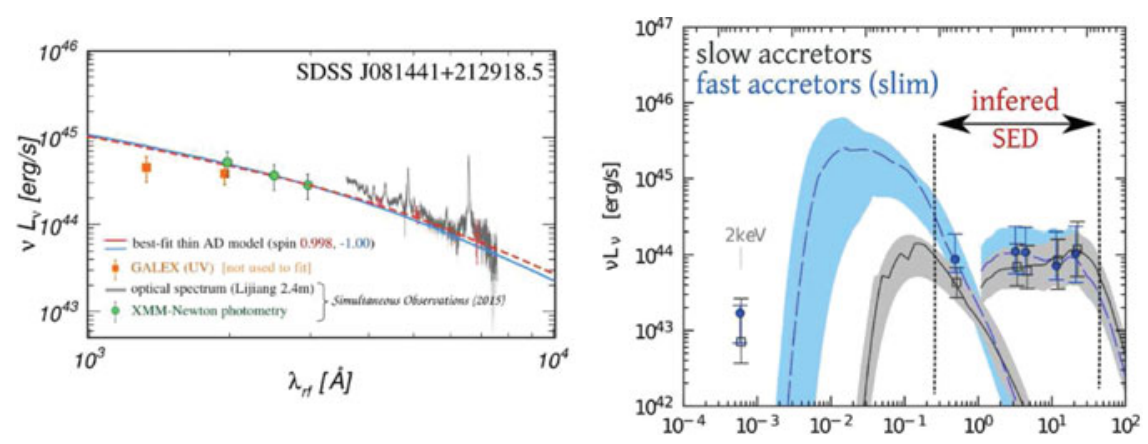

Figure 3. Left: An example for thin accretion disk model fitted to the data of the intrinsic $0.2-1 \mu \mathrm{m}$ SEAMBH SED. Right: The median SED for sub-Eddington and supper-Eddington sources show no statistical evidence for different optical/UV spectrum or torus emission. See Castello-Mor, Netzer \& Kaspi (2016) for more details.

spectral energy distribution of slim discs is rather uncertain and raise several important questions for high accretion rate AGN (e.g. Sadowsky \& Narayan 2015; Wang et al. 2015).

To test whether thin accretion disk can fit the optical/UV spectral energy distribution (SED) of SEAMBHs we used a RM sample of both SEAMBHs and normal AGNs and fitted them with a model of thin accretion disk. The fitting takes into account the measured $\mathrm{BH}$ mass, accretion rate, $\mathrm{BH}$ spin, and intrinsic reddening of the sources. This study was published in Castello-Mor, Netzer \& Kaspi (2016) and Castello-Mor et al. (2017). We found that thin accretion disk models can be used to obtain a satisfactory fits to the intrinsic 0.2-1 $\mu \mathrm{m}$ SEAMBH SED (Fig. 3 left). The median SED for sub-Eddington and supper-Eddington sources show no statistical evidence for different optical/UV spectrum or torus emission.

We found Various indications for transition in X-ray and UV properties at AGNs with very high Eddington ratio. Additional physics, occurring in the nuclear regions of SEAMBHs and responsible for making the slim disk SED consistent with that of thin disk, must be at work. One option can be that powerful winds can carry away a significant amount of the accreted energy from the innermost regions, decreasing its UV radiation and perhaps quenching the disk corona.

\section{References}

Bentz, M., et al. 2013, ApJ, 127, 67

Bentz, M. \& Katz, S. 2015, PASP, 767, 149

Castell-Mor, N., Netzer, H., \& Kaspi, S. 2016, MNRAS, 458, 1839

Castell-Mor, N., et al. 2017, MNRAS, submitted

Kaspi, S., Brandt, W. H., Maoz, D., Netzer, H., Schneider, D.P., \& Shemmer O. 2007, ApJ,659, 997

Kaspi, S., Smith, P. S., Netzer, H., Maoz, D., Jannuzi, B., \& Giveon, U. 2000, ApJ, 533, 631

Du, P., et al. 2014, ApJ, 782, 45

Du, P., et al. 2015, ApJ, 806, 22

$\mathrm{Hu}, \mathrm{C}$, et al. 2015, ApJ, 804, 138

Peterson, B. M. 1993, PASP, 105, 247

Peterson, B. M., et al. 2005, ApJ, 632, 799 (erratum 641, 638 [2006])

Sadowski, A. 2011, Ph.D. thesis, arXiv:1108.0396

Sadowski, A, Narayan, R. 2015, MNRAS, 454, 2372

Vestergaard, M., Fan, X., Tremonti, C. A., Osmer, P. S., \& Rechards, G. T. 2008, ApJL, 674, L1

Wang, J.-M., et al. 2014, ApJ, 793, 108 